\title{
On Fourth and Fifth Order Explicit Almost Runge - Kutta Methods
}

\begin{abstract}
Abdulrahman Ndanusa
Department of Mathematics

Federal University of Technology Minna, Nigeria

as.ndanusa@futminna.edu.ng,

asndanusa@yahoo.com

\author{
Khadeejah James Audu \\ Department of Mathematics
}

Federal University of Technology Minna, Nigeria jameskhadeejah@yahoo.com

Abstract: Almost Runge - Kutta (ARK) methods are a special class of general linear methods that have close affinity to Runge - Kutta methods for the numerical integration of initial value problems of ordinary differential equations, but with some advantages over the classical Runge - Kutta methods. In this research work, we exploit the order, annihilation and Runge - Kutta stability conditions normally associated with Runge - Kutta methods to derive two new explicit Almost Runge - Kutta methods of orders four (ARK4) and five (ARK5)respectively. The methods were tested for consistency and stability and proven to satisfy the criteria for both; hence their convergence is guaranteed. In order to demonstrate the efficiency and reliability of the proposed methods, some initial value problems were solved with the methods. Numerical results revealed that the proposed methods exhibited numerical errors within acceptable limits when compared with the exact solutions. More so, the methods performed better than some existing Almost Runge - Kutta methods of equal standing.
\end{abstract}

Keywords: Initial value problem, Order condition, Convergence, Stability, Consistency.

\section{INTRODUCTION}

In the quest to achieve an overall general formation of numerical schemes that combine the multivalued features of linear multistep methods along with the multistage features of Runge-Kutta methods, Butcher [1] introduced the general linear methods. In general linear methods the multistage features of Runge-Kutta schemes are linked with the perception of passing more values in- between steps. Almost Runge-Kutta (ARK) methods are a special type of general linear methods whose properties closely resemble those of explicit Runge-Kutta methods. They were introduced by Butcher [2] for the purpose of preserving the multi-stage character of Runge-Kutta scheme as well as passing many values between steps, thereby giving the method a multi-value character [3]. They general ARK method takes the form:

$$
\left[\begin{array}{c}
Y_{1} \\
Y_{2} \\
\vdots \\
Y_{s} \\
- \\
y_{1}^{[n]} \\
y_{2}^{[n]} \\
y_{r}^{[n]}
\end{array}\right]=\left[\begin{array}{l|l}
A & U \\
\hline B & V
\end{array}\right]\left[\begin{array}{cc}
h f & Y_{1} \\
h f & Y_{2} \\
\vdots \\
h f & Y_{s} \\
-- \\
y_{1}^{[n-1]} \\
y_{2}^{[n-1]} \\
y_{r}^{[n-1]}
\end{array}\right]
$$

where $s$ is the number of internal stages. For an order $p$ method the three output values are

$$
\left.\begin{array}{c}
y_{1}^{[n]}=y\left(x_{n}\right)+o\left(h^{p+1}\right), \\
y_{2}^{[n]}=h y^{\prime}\left(x_{n}\right)+o\left(h^{p+1}\right), \\
y_{3}^{[n]}=h^{3} y^{n}\left(x_{n}\right)+o\left(h^{3}\right)
\end{array}\right\}
$$


The coefficients of the method are chosen in a careful way to ensure the simple stability properties of Runge-Kutta methods are retained.

In this research we will concentrate on methods where $A$ is strictly lower triangular, and hence the method is explicit. The general form of an explicit ARK method is

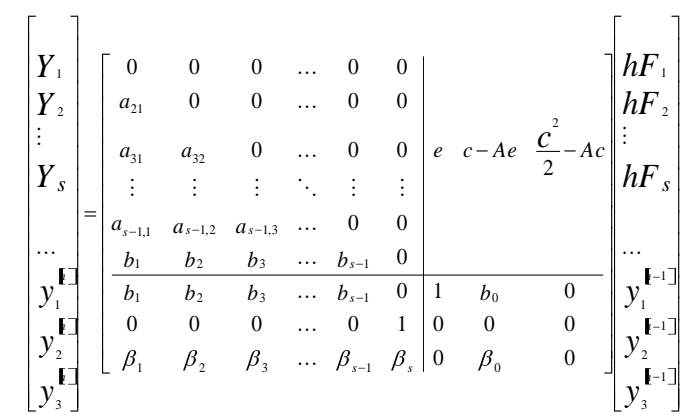

where $Y_{1}, Y_{2}, \ldots n, Y_{s}$ are the Internal stages, $y_{1}^{[n]}, y_{2}^{[[n]}$ and $y_{3}^{[n]}$ are the Output approximations, $y_{1}^{[n-1]} y_{2}^{[n-1]} y_{3}^{[n-1]}$ are the Input approximations and the stage derivatives are $F_{1}, F_{2}, \ldots, F_{z}$, where $F_{i}=f\left(x, Y_{i}\right)$. Just like in the case of Runge-Kutta methods, $b$ is a vector of length $s$ representing the weights and $c$ is a vector of length $s$ representing the positions at which function $f$ is evaluated. The vector $e$ is a unit vector of length $s$ consisting of entirely $1 \mathrm{~s}$.

In this paper, we seek to derive two explicit ARK methods of orders four and five with four and five stages respectively (i.e., methods with $s=p=4$ and $s=p=5$ ).

\section{METHODS}

\subsection{Derivation of ARK4 $(s=p=4)$}

The general fourth order four stages scheme is given as :

$$
\left[\begin{array}{ll}
A & U \\
\hline B & V
\end{array}\right]=\left[\begin{array}{cccc|ccc}
0 & 0 & 0 & 0 & 1 & c_{1} & \frac{1}{2} c_{1}^{2} \\
a_{21} & 0 & 0 & 0 & 1 & c_{2}-a_{21} & \frac{1}{2} c_{2}^{2}-a_{21} c_{1} \\
a_{31} & a_{32} & 0 & 0 & 1 & c_{3}-a_{31}-a_{32} & \frac{1}{2} c_{3}^{2}-a_{31} c_{1}-a_{32} c_{2} \\
b_{1} & b_{2} & b_{3} & 0 & 1 & b_{0} & 0 \\
\hline b_{1} & b_{2} & b_{3} & 0 & 1 & b_{0} & 0 \\
0 & 0 & 0 & 1 & 0 & 0 & 0 \\
\beta_{1} & \beta_{2} & \beta_{3} & \beta_{4} & 0 & \beta_{0} & 0
\end{array}\right]
$$

The abscissa vector $c=\left[c_{1}, c_{2}, c_{3}, 1\right]^{T}, b^{T}=\left[b_{1}, b_{2}, b_{3}, 0\right], \beta^{T}=\left[\beta_{1}, \beta_{2}, \beta_{3}, \beta_{4}\right]$. The constituents of the first output approximations for order four with four stages are given below:

$$
\begin{gathered}
b_{0}+b^{T} e=1 \\
b^{T} c=\frac{1}{2} \\
b^{T} c^{2}=\frac{1}{3} \\
b^{T} c^{3}=\frac{1}{4} \\
b^{T} A c^{2}=\frac{1}{12} \\
b^{T}\left(\frac{1}{2} c^{2}-A c\right)=0
\end{gathered}
$$


Combining (7) and (10) we obtain

$$
b^{T} A c=\frac{1}{6}
$$

Either equation (10) or the same condition in (11) will be designated as 'annihilation conditions'. The vectors $\beta^{T}$ and $\beta_{0}$, represent the $3^{\text {rd }}$ outgoing estimation, which is meant to produce the result $h^{2} y^{n}\left(x_{n+1}\right)+o\left(h^{3}\right)$. It follows that,

$$
\begin{gathered}
\beta^{T} e+\beta_{0}=0 \\
\beta^{T} c=1
\end{gathered}
$$

The Runge-Kutta stability conditions are,

$$
\begin{gathered}
\beta^{T}\left(I+\beta_{4} A\right)=\beta_{4} e_{4}^{T} \\
c_{1}=-2 \frac{\exp _{4}\left(-\beta_{8}\right)}{\beta_{4} \exp _{3}\left(-\beta_{4}\right)} \\
\left(1+\frac{1}{2} \beta_{4} c_{1}\right) b^{T} A^{2} c=\frac{1}{4 !}
\end{gathered}
$$

From (15), it follows that

$$
c_{1}=-\frac{2\left(1-\beta_{4}+\frac{1}{2} \beta_{4}^{2}-\frac{1}{6} \beta_{4}^{3}+\frac{1}{24} \beta_{4}^{4}\right)}{\beta_{4}\left(1-\beta_{4}+\frac{1}{2} \beta_{4}^{2}-\frac{1}{6} \beta_{4}^{3}\right)}
$$

From (5) - (8), we obtained

$$
\left.\begin{array}{l}
b_{0}+b_{1}+b_{2}+b_{3}=1 \\
b_{1} c_{1}+b_{2} c_{2}+b_{3} c_{3}=\frac{1}{2} \\
b_{1} c_{1}^{2}+b_{2} c_{2}^{2}+b_{3} c_{3}^{2}=\frac{1}{3} \\
b_{1} c_{1}^{3}+b_{2} c_{2}^{3}+b_{3} c_{3}^{3}=\frac{1}{4}
\end{array}\right\}
$$

Thus,

$$
\begin{array}{r}
b_{1}=\frac{6 c_{2} c_{3}-4 c_{2}-4 c_{3}+3}{12 c_{1}\left(c_{1}-c_{3}\right)\left(c_{1}-c_{2}\right)} \\
b_{2}=\frac{6 c_{1} c_{3}-4 c_{1}-4 c_{3}+3}{12 c_{2}\left(c_{1}-c_{2}\right)\left(c_{2}-c_{3}\right)} \\
b_{3}=\frac{6 c_{1} c_{2}-4 c_{1}-4 c_{2}+3}{12 c_{3}\left(c_{2}-c_{3}\right)\left(c_{1}-c_{3}\right)} \\
b_{0}=\frac{12 c_{1} c_{2} c_{3}-6 c_{1} c_{2}-6 c_{2} c_{3}+4 c_{1}+4 c_{2}+4 c_{3}-3}{12 c_{1} c_{2} c_{3}}
\end{array}
$$

And,

$$
\begin{array}{r}
a_{21}=\frac{1}{12 b_{3} a_{32} c_{1}\left(2+\beta_{4} c_{1}\right)} \\
a_{31}=\frac{\frac{1}{6}-b_{3} a_{32} c_{2}-b_{2} a_{21} c_{1}}{b_{3} c_{1}} \\
a_{32}=\frac{1-2 c_{1}}{12 b_{3} c_{2}\left(c_{2}-c_{1}\right)}
\end{array}
$$

From (12) and (14) we evaluate the remaining values of $\beta^{T}$ vector $\beta_{0}$. Eventually the following ARK4 method is obtained. 
ARK4 with $c^{T}=\left[\frac{15}{34}, \frac{1}{2}, 1,1\right], \beta_{4}=4$

2.2 Derivation of ARK5 $(s=p=5)$

$$
\left[\begin{array}{l|l}
A & U \\
B & V
\end{array}\right]=\left[\begin{array}{cccc|ccc}
0 & 0 & 0 & 0 & 1 & \frac{15}{34} & \frac{225}{2312} \\
\frac{289}{1920} & 0 & 0 & 0 & 1 & \frac{671}{1920} & \frac{15}{256} \\
-\frac{289}{480} & 2 & 0 & 0 & 1 & -\frac{191}{480} & -\frac{15}{64} \\
0 & \frac{2}{3} & \frac{1}{6} & 0 & 1 & \frac{1}{6} & 0 \\
\hline 0 & \frac{2}{3} & \frac{1}{6} & 0 & 1 & \frac{1}{6} & 0 \\
0 & 0 & 0 & 1 & 0 & 0 & 0 \\
-\frac{578}{45} & \frac{32}{3} & -\frac{8}{3} & 4 & 0 & -\frac{38}{45} & 0
\end{array}\right]
$$

The general fifth order, five stages scheme is:

$$
\left[\begin{array}{ll}
A & U \\
B & V
\end{array}\right]=\left[\begin{array}{ccccc|ccc}
0 & 0 & 0 & 0 & 0 & 1 & c_{1} & \frac{1}{2} c_{1}^{2} \\
a_{21} & 0 & 0 & 0 & 0 & 1 & c_{2}-a_{21} & \frac{1}{2} c_{2}^{2}-a_{21} c_{1} \\
a_{31} & a_{32} & 0 & 0 & 0 & 1 & c_{3}-a_{31}-a_{32} & \frac{1}{2} c_{3}^{2}-a_{31} c_{1}-a_{32} c_{2} \\
a_{41} & a_{42} & a_{43} & 0 & 0 & 1 & c_{4}-a_{41}-a_{42}-a_{43} & \frac{1}{2} c_{4}^{2}-a_{41} c_{1}-a_{42} c_{2}-a_{43} c_{3} \\
b_{1} & b_{2} & b_{3} & b_{4} & 0 & 1 & b_{0} & 0 \\
\hline b_{1} & b_{2} & b_{3} & b_{4} & 0 & 1 & b_{0} & 0 \\
0 & 0 & 0 & 0 & 1 & 0 & 0 & 0 \\
\beta_{1} & \beta_{2} & \beta_{3} & \beta_{4} & \beta_{5} & 0 & \beta_{0} & 0
\end{array}\right]
$$

where $c_{5}=1$. The Order conditionsare:

$$
\begin{gathered}
b^{T} c=\frac{1}{2} \\
b^{T} c^{2}=\frac{1}{3} \\
b^{T} c^{3}=\frac{1}{4} \\
b^{T} c^{4}=\frac{1}{5} \\
b_{0}+b^{T} e=1 \\
b^{T} A=b^{T}(1-C) \\
b^{T}(1-c) A c=\frac{1}{24} \\
b^{T}(1-c) A c^{2}=\frac{1}{60} \\
c_{1}=-2 \frac{\exp _{5}\left(-\beta_{5}\right)}{\beta_{5} \exp _{4}\left(-\beta_{5}\right)} \\
\beta^{T} e+\beta_{0}=0 \\
\beta^{T}\left(I+\beta_{5} A\right)=\beta_{5} e_{5}^{T} \\
\left(1+\frac{1}{2} \beta_{5} c_{1}\right) b^{T} A^{3} c=\frac{1}{120}
\end{gathered}
$$


We have 3 free parameters designated as $c_{2}, c_{3}$ and $\beta_{5}$. Thus,

$$
\begin{aligned}
& c_{1}=\frac{-2\left(1-\beta_{5}+\frac{1}{2} \beta_{5}^{2}-\frac{1}{6} \beta_{5}^{3}+\frac{1}{24} \beta_{5}^{4}+\frac{1}{120} \beta_{5}^{5}\right)}{\beta_{5}\left(1-\beta_{5}+\frac{1}{2} \beta_{5}^{2}-\frac{1}{6} \beta_{5}^{3}+\frac{1}{24} \beta_{5}^{4}\right)} \\
& a_{32}=\frac{2-5 c_{1}}{120 b_{3}\left(c_{2}^{2}-c_{1} c_{2}-c_{2}^{2} c_{3}+c_{1} c_{2} c_{3}\right)} \\
& a_{31}=\frac{\frac{1}{24}-b_{2} a_{21} c_{1}\left(1-c_{2}\right)-b_{3} a_{32} c_{2}\left(1-c_{3}\right)}{b_{3} c_{1}\left(1-c_{3}\right)} \\
& a_{21}=\frac{2 c_{2}\left(c_{2}-c_{1}\right)}{c_{1}\left(2-5 c_{1}\right)\left(2+c_{1} \beta_{5}\right)} \\
& a_{41}=\frac{b_{1}-b_{1} c_{1}-b_{2} a_{32}-b_{3} a_{31}}{b_{4}} \\
& a_{42}=\frac{b_{1}-b_{2} c_{2}-b_{3} a_{32}}{b_{4}} \\
& a_{43}=\frac{b_{3}-b_{3} c_{3}}{b_{3}} \\
& b_{1} c_{1}+b_{2} c_{2}+b_{3} c_{3}+b_{4} c_{4}=\frac{1}{2} \\
& b_{1} c_{1}+b_{2} c_{2}+b_{3} c_{3}+b_{4} c_{4}=\frac{1}{2} \\
& \left.b_{1} c_{1}^{3}+b_{2} c_{2}^{3}+b_{3} c_{3}^{3}+b_{4} c_{4}^{3}=\frac{1}{4}\right\} \\
& b_{1} c_{1}^{4}+b_{2} c_{2}^{4}+b_{3} c_{3}^{4}+b_{4} c_{4}^{4}=\frac{1}{5} \\
& b_{0}+b_{1}+b_{2}+b_{3}+b_{4}=1 \\
& b_{1}=\frac{5 c_{2}+5 c_{3}-10 c_{2} c_{3}-3}{60\left(c_{1}-1\right) c_{1}\left(c_{1}-c_{2}\right)\left(c_{1}-c_{3}\right)} \\
& b_{2}=\frac{10 c_{1} c_{3}-5 c_{1}-5 c_{3}+3}{60 c_{2}\left(c_{1}-c_{2}\right)\left(c_{2}-1\right)\left(c_{2}-c_{3}\right)} \\
& b_{3}=-\frac{1}{60}\left[\frac{10 c_{1} c_{2}-5 c_{1}-5 c_{2}+3}{c_{3}\left(c_{3}-1\right)\left(c_{2}-c_{3}\right)\left(c_{1}-c_{3}\right)}\right] \\
& b_{4}=\frac{30 c_{1} c_{2} c_{3}-20 c_{1} c_{2}-20 c_{1} c_{3}-20 c_{2} c_{3}+15 c_{1}+15 c_{2}+15 c_{3}-c_{2}}{60\left(c_{3}-1\right)\left(c_{2}-1\right)\left(c_{1}-1\right)} \\
& b_{0}=\frac{30 c_{1} c_{2} c_{3}-10 c_{1} c_{2}-10 c_{1} c_{3}-10 c_{2} c_{3}+5 c_{1}+5 c_{2}+5 c_{3}-3}{60 c_{1} c_{2} c_{3}}
\end{aligned}
$$

After obtaining the remaining elements of $\beta^{T}$ we obtain the following ARK5 scheme.

ARK5 with $c^{T}=\left[\frac{52}{165}, \frac{1}{3}, \frac{3}{4}, 1,1\right]$

$$
\left[\frac{A}{B} \mid \begin{array}{l}
U \\
\hline
\end{array}\right]=\left[\begin{array}{ccccc|ccc}
0 & 0 & 0 & 0 & 0 & 1 & \frac{52}{165} & \frac{1352}{27225} \\
\frac{605}{19656} & 0 & 0 & 0 & 0 & 1 & \frac{5947}{19656} & \frac{26}{567} \\
-\frac{768955}{159744} & \frac{6027}{1024} & 0 & 0 & 0 & 1 & -\frac{51449}{519744} & -\frac{377}{2304} \\
\frac{1802767635}{51845976} & -\frac{7006}{193} & \frac{231424}{166173} & 0 & 0 & 1 & \frac{1388045}{1264536} & \frac{21346}{36477} \\
\frac{5490375}{6745648} & -\frac{3}{8} & \frac{1024}{2583} & \frac{193}{2712} & 0 & 1 & \frac{175}{1872} & 0 \\
\frac{5490375}{6745648} & -\frac{3}{8} & \frac{1024}{2583} & \frac{193}{2712} & 0 & 1 & \frac{175}{1872} & 0 \\
0 & 0 & 0 & 0 & 1 & 0 & 0 & 0 \\
\frac{34641995}{6745648} & -\frac{405}{8} & -\frac{256}{287} & -\frac{579}{904} & 3 & 0 & -\frac{457}{208} & 0
\end{array}\right]
$$




\subsection{Convergence Analysis of ARK4}

The matrix $V$ of the ARK4 scheme of (26)must have bounded power for the method to be stable. Thus

$$
V=\left[\begin{array}{ccc}
1 & \frac{1}{6} & 0 \\
0 & 0 & 0 \\
0 & -\frac{38}{45} & 0
\end{array}\right]
$$

The characteristic polynomial of $V$ is

$$
\rho(\lambda)=\lambda^{3}-\lambda^{2}
$$

And the eigenvalues are found to be $\lambda_{1}=1, \lambda_{2}=0, \lambda_{3}=0$. By Cayley-Hamilton theorem,

$$
\begin{gathered}
\rho(V)=V^{3}-V^{2}=0 \\
\left\lfloor\begin{array}{lll}
1 & \frac{1}{6} & 0 \\
0 & 0 & 0 \\
0 & 0 & 0
\end{array}\right\rfloor-\left[\begin{array}{lll}
1 & \frac{1}{6} & 0 \\
0 & 0 & 0 \\
0 & 0 & 0
\end{array}\right\rfloor=\left[\begin{array}{lll}
0 & 0 & 0 \\
0 & 0 & 0 \\
0 & 0 & 0
\end{array}\right]
\end{gathered}
$$

This implies that

$$
V^{3}=V^{2}
$$

Similarly, $V^{4}-V^{2}=0$, implies $V^{4}=V^{2}, V^{5}-V^{2}=0$, implies $V^{5}=V^{2}, \ldots V^{n}=V^{2}$, for every $n$ greater than 2 ; it implies $V^{n}$ is bounded and thus the method is stable. The method is equally consistent since it is of order $p=4>1$; hence its convergence.

\subsection{Convergence Analysis of ARK5}

The matrix $V$ of ARK5 scheme (53) is

$$
V=\left[\begin{array}{ccc}
1 & \frac{175}{1872} & 0 \\
0 & 0 & 0 \\
0 & -\frac{457}{208} & 0
\end{array}\right]
$$

The eigenvalues of (59) calculated to be 1, 0, and 0. By Cayley-Hamilton theorem the characteristic equation is

$$
\rho(V)=V^{3}-V^{2}=0
$$

This implies that $V^{3}=V^{2}$. Similarly, $V^{4}-V^{2}=0$, implies $V^{4}=V^{2}, V^{5}-V^{2}=0$, implies $V^{5}=V^{2}{ }_{y} \ldots V^{n}=V^{2}$, for every $n$ greater than 2; it implies $V^{n}$ is bounded and thus the method is stable. The ARK5 method is also consistent since it is of order $p=5>1$. Therefore it is convergent.

\section{RESUlTS AND DisCUSSION}

The following problems are solved with the derived schemes.

Problem 1:

$$
\left.\begin{array}{cc}
y^{y}=x+y, & y(0)=1 \\
h=0.1, & x \in[0,2] \\
\text { Exact solution: } & y_{E}(x)=2 e^{x}-x-1
\end{array}\right\}
$$

This problem is found in [4].

Problem 2:

$$
\begin{aligned}
& \left.\begin{array}{c}
y^{y}=\frac{y(x)}{4}\left(1-\frac{y(x)}{20}\right), \quad y(0)=1 \\
h=0.1, \quad x \in[0,2]
\end{array}\right\} \\
& \text { Exact solution: } \left.y_{E}(x)=\frac{20}{1+19 e^{\frac{-1}{4} x}}\right\}
\end{aligned}
$$


This problem is found in [3] and [5].

Problem 3:

$$
\left.\begin{array}{c}
y^{y}=\frac{y+x}{y-x}, \quad y(0)=1 \\
h=0.1, \quad 0 \leq x \leq 2 \\
\text { t solution: } \quad y_{E}(x)=x+\sqrt{1+2 x^{2}}
\end{array}\right\}
$$

This problem is found in [6].

Problem 1, 2 and 3 solved using the derived schemes (ARK4 and ARK5). The results are compared with methods of appropriate orders derived by [3], [5] and [7]. The results are presented as follows.

Table 1. Comparison of results (ARK4, Problem 1)

\begin{tabular}{|l|c|l|l|l|l|}
\hline $\boldsymbol{x}$ & $\boldsymbol{y}_{\text {exact }}$ & $\boldsymbol{y}_{\text {approx }[\text { ARK4 }]}$ & Error $_{\text {ARK4 }}$ & $\boldsymbol{y}_{\left.\text {approx }[]_{1}\right]}$ & Error $_{\left.[]_{1}\right]}$ \\
\hline $\mathbf{0 . 0}$ & 1.0000000000 & 1.0000000000 & $0.0000000000 \mathrm{E}+00$ & 1.00000000000 & $0.0000000000 \mathrm{E}+00$ \\
\hline $\mathbf{0 . 1}$ & 1.1103418362 & 1.1103417643 & $7.1899999954 \mathrm{E}-08$ & 1.1111474306 & $8.0559440000 \mathrm{E}-04$ \\
\hline $\mathbf{0 . 2}$ & 1.2428055163 & 1.2428052496 & $2.6670000008 \mathrm{E}-07$ & 1.2446576379 & $1.8521216000 \mathrm{E}-03$ \\
\hline $\mathbf{0 . 3}$ & 1.3997176152 & 1.3997171134 & $5.0180000000 \mathrm{E}-07$ & 1.4029079395 & $3.1903243000 \mathrm{E}-03$ \\
\hline $\mathbf{0 . 4}$ & 1.5836493953 & 1.5836486120 & $7.8329999997 \mathrm{E}-07$ & 1.5885194451 & $4.8700498000 \mathrm{E}-03$ \\
\hline $\mathbf{0 . 5}$ & 1.7974425414 & 1.7974414229 & $1.1185000000 \mathrm{E}-06$ & 1.8043910308 & $6.9484894000 \mathrm{E}-03$ \\
\hline $\mathbf{0 . 6}$ & 2.0442376008 & 2.0442360852 & $1.5155999997 \mathrm{E}-06$ & 2.0537287033 & $9.4911025000 \mathrm{E}-03$ \\
\hline $\mathbf{0 . 7}$ & 2.3275054149 & 2.3275034311 & $1.9838000003 \mathrm{E}-06$ & 2.3400781409 & $1.2572726000 \mathrm{E}-02$ \\
\hline $\mathbf{0 . 8}$ & 2.6510818570 & 2.6510793232 & $2.5338000000 \mathrm{E}-06$ & 2.6673606805 & $1.6278823500 \mathrm{E}-02$ \\
\hline $\mathbf{0 . 9}$ & 3.0192062223 & 3.0192030449 & $3.1774000000 \mathrm{E}-06$ & 3.0399131186 & $2.0706896300 \mathrm{E}-02$ \\
\hline $\mathbf{1 . 0}$ & 3.4365636569 & 3.4365597285 & $3.9284000004 \mathrm{E}-06$ & 3.4625317281 & $2.5968071200 \mathrm{E}-02$ \\
\hline $\mathbf{1 . 1}$ & 3.9083320479 & 3.9083272456 & $4.8022999999 \mathrm{E}-06$ & 3.9405209395 & $3.2188891600 \mathrm{E}-02$ \\
\hline $\mathbf{1 . 2}$ & 4.4402338455 & 4.4402280289 & $5.8165999999 \mathrm{E}-06$ & 4.4797471790 & $3.9513333500 \mathrm{E}-02$ \\
\hline $\mathbf{1 . 3}$ & 5.0385933352 & 5.0385863443 & $6.9909000002 \mathrm{E}-06$ & 5.0866984113 & $4.8105076100 \mathrm{E}-02$ \\
\hline $\mathbf{1 . 4}$ & 5.7103999337 & 5.7103915856 & $8.3480999997 \mathrm{E}-06$ & 5.7685499907 & $5.8150057000 \mathrm{E}-02$ \\
\hline $\mathbf{1 . 5}$ & 6.4633781407 & 6.4633682273 & $9.9133999996 \mathrm{E}-06$ & 6.5332374885 & $6.9859347800 \mathrm{E}-02$ \\
\hline $\mathbf{1 . 6}$ & 7.3060648488 & 7.3060531332 & $1.1715600000 \mathrm{E}-05$ & 7.3895372371 & $8.3472388300 \mathrm{E}-02$ \\
\hline $\mathbf{1 . 7}$ & 8.2478947835 & 8.2478809963 & $1.3787200000 \mathrm{E}-05$ & 8.3471554074 & $9.9260623900 \mathrm{E}-02$ \\
\hline $\mathbf{1 . 8}$ & 9.2992949288 & 9.2992787639 & $1.6164900000 \mathrm{E}-05$ & 9.4168265232 & $1.1753159440 \mathrm{E}-01$ \\
\hline $\mathbf{1 . 9}$ & 1.0000000000 & 1.0000000000 & $1.8891000000 \mathrm{E}-05$ & 10.6104224140 & $1.3863352900 \mathrm{E}-01$ \\
\hline $\mathbf{2 . 0}$ & 1.1103418362 & 1.1103417643 & $2.2010000000 \mathrm{E}-05$ & 11.9410727110 & $1.6296051300 \mathrm{E}-01$ \\
\hline
\end{tabular}

Table 2. Comparison of results (ARK4, Problem 2)

\begin{tabular}{|l|c|l|l|l|l|}
\hline $\boldsymbol{x}$ & $y_{\text {exact }}$ & $y_{\text {approx }[A R K 4]}$ & Error $_{\text {ARK4 }}$ & $y_{\text {approx [5] }}$ & Error $_{[5]}$ \\
\hline $\mathbf{0 . 0}$ & 1.0000000000 & 1.0000000000 & $0.0000000000 \mathrm{E}+00$ & 1.0000000000 & $0.0000000000 \mathrm{E}+00$ \\
\hline $\mathbf{0 . 1}$ & 1.0240189623 & 1.0240189623 & $0.0000000000 \mathrm{E}+00$ & 1.0215450040 & $2.4739583000 \mathrm{E}-03$ \\
\hline $\mathbf{0 . 2}$ & 1.0485829964 & 1.0485829963 & $1.0000000830 \mathrm{E}-10$ & 1.0435322524 & $5.0507440000 \mathrm{E}-03$ \\
\hline $\mathbf{0 . 3}$ & 1.0737029289 & 1.0737029287 & $2.0000001650 \mathrm{E}-10$ & 1.0659660257 & $7.7369032000 \mathrm{E}-03$ \\
\hline $\mathbf{0 . 4}$ & 1.0993897267 & 1.0993897266 & $1.0000000830 \mathrm{E}-10$ & 1.0888542255 & $1.0535501200 \mathrm{E}-02$ \\
\hline $\mathbf{0 . 5}$ & 1.1256544953 & 1.1256544951 & $2.0000001650 \mathrm{E}-10$ & 1.1122048501 & $1.3449645200 \mathrm{E}-02$ \\
\hline $\mathbf{0 . 6}$ & 1.1525084759 & 1.1525084756 & $3.0000002480 \mathrm{E}-10$ & 1.1360259870 & $1.6482488900 \mathrm{E}-02$ \\
\hline $\mathbf{0 . 7}$ & 1.1799630432 & 1.1799630429 & $2.9999980280 \mathrm{E}-10$ & 1.1603258102 & $1.9637233000 \mathrm{E}-02$ \\
\hline $\mathbf{0 . 8}$ & 1.2080297028 & 1.2080297024 & $4.0000003310 \mathrm{E}-10$ & 1.1851125795 & $2.2917123300 \mathrm{E}-02$ \\
\hline $\mathbf{0 . 9}$ & 1.2367200876 & 1.2367200871 & $5.0000004140 \mathrm{E}-10$ & 1.2103946386 & $2.6325449000 \mathrm{E}-02$ \\
\hline $\mathbf{1 . 0}$ & 1.2660459552 & 1.2660459547 & $5.0000004140 \mathrm{E}-10$ & 1.2361804129 & $2.9865542300 \mathrm{E}-02$ \\
\hline $\mathbf{1 . 1}$ & 1.2960191836 & 1.2960191829 & $6.9999983590 \mathrm{E}-10$ & 1.2624784081 & $3.3540775500 \mathrm{E}-02$ \\
\hline $\mathbf{1 . 2}$ & 1.3266517673 & 1.3266517667 & $6.0000004960 \mathrm{E}-10$ & 1.2892972073 & $3.7354560000 \mathrm{E}-02$ \\
\hline $\mathbf{1 . 3}$ & 1.3579558136 & 1.3579558128 & $8.0000006620 \mathrm{E}-10$ & 1.3166454694 & $4.1310344200 \mathrm{E}-02$ \\
\hline $\mathbf{1 . 4}$ & 1.3899435370 & 1.3899435363 & $7.0000005790 \mathrm{E}-10$ & 1.3445319259 & $4.5411611100 \mathrm{E}-02$ \\
\hline $\mathbf{1 . 5}$ & 1.4226272553 & 1.4226272544 & $8.9999985240 \mathrm{E}-10$ & 1.3729653789 & $4.9661876400 \mathrm{E}-02$ \\
\hline $\mathbf{1 . 6}$ & 1.4560193830 & 1.4560193821 & $8.9999985240 \mathrm{E}-10$ & 1.4019546976 & $5.4064685400 \mathrm{E}-02$ \\
\hline $\mathbf{1 . 7}$ & 1.4901324269 & 1.4901324258 & $1.1000000910 \mathrm{E}-09$ & 1.4315088158 & $5.8623611100 \mathrm{E}-02$ \\
\hline $\mathbf{1 . 8}$ & 1.5249789787 & 1.5249789776 & $1.1000000910 \mathrm{E}-09$ & 1.4616367283 & $6.3342250400 \mathrm{E}-02$ \\
\hline $\mathbf{1 . 9}$ & 1.5605717097 & 1.5605717086 & $1.1000000910 \mathrm{E}-09$ & 1.4923474874 & $6.8224222300 \mathrm{E}-02$ \\
\hline $\mathbf{2 . 0}$ & 1.5969233630 & 1.5969233618 & $1.1999998770 \mathrm{E}-09$ & 1.5236501996 & $7.3273163400 \mathrm{E}-02$ \\
\hline
\end{tabular}


Table 3. Comparison of results (ARK5, Problem 2)

\begin{tabular}{|l|c|l|l|l|l|}
\hline $\boldsymbol{x}$ & $y_{\text {exact }}$ & $y_{\text {approx }}$ ARK 4$]_{]}$ & \multicolumn{1}{|c|}{ Error $_{\text {ARK4 }}$} & $y_{\text {approx }[7]}$ & Error $_{[7]}$ \\
\hline $\mathbf{0 . 0}$ & 1.0000000000 & 1.0000000000 & $0.0000000000 \mathrm{E}+00$ & 1.0000000000 & $0.0000000000 \mathrm{E}+00$ \\
\hline $\mathbf{0 . 1}$ & 1.0240189623 & 1.0240189624 & $1.0000000827 \mathrm{E}-10$ & 1.0240183659 & $5.9640000005 \mathrm{E}-07$ \\
\hline $\mathbf{0 . 2}$ & 1.0485829964 & 1.0485829964 & $0.0000000000 \mathrm{E}+00$ & 1.0484502786 & $1.3271780000 \mathrm{E}-04$ \\
\hline $\mathbf{0 . 3}$ & 1.0737029289 & 1.0737029288 & $1.0000000827 \mathrm{E}-10$ & 1.0734007162 & $3.0221270000 \mathrm{E}-04$ \\
\hline $\mathbf{0 . 4}$ & 1.0993897267 & 1.0993897267 & $0.0000000000 \mathrm{E}+00$ & 1.0989036548 & $4.8607190000 \mathrm{E}-04$ \\
\hline $\mathbf{0 . 5}$ & 1.1256544953 & 1.1256544953 & $0.0000000000 \mathrm{E}+00$ & 1.1249757264 & $6.7876890000 \mathrm{E}-04$ \\
\hline $\mathbf{0 . 6}$ & 1.1525084759 & 1.1525084759 & $0.0000000000 \mathrm{E}+00$ & 1.1516294190 & $8.7905690000 \mathrm{E}-04$ \\
\hline $\mathbf{0 . 7}$ & 1.1799630432 & 1.1799630432 & $0.00000000000 \mathrm{E}+00$ & 1.1788763098 & $1.0867334000 \mathrm{E}-03$ \\
\hline $\mathbf{0 . 8}$ & 1.2080297028 & 1.2080297028 & $0.0000000000 \mathrm{E}+00$ & 1.2067278538 & $1.3018490000 \mathrm{E}-03$ \\
\hline $\mathbf{0 . 9}$ & 1.2367200876 & 1.2367200876 & $0.0000000000 \mathrm{E}+00$ & 1.2351955743 & $1.5245133000 \mathrm{E}-03$ \\
\hline $\mathbf{1 . 0}$ & 1.2660459552 & 1.2660459552 & $0.00000000000 \mathrm{E}+00$ & 1.2642911058 & $1.7548494000 \mathrm{E}-03$ \\
\hline $\mathbf{1 . 1}$ & 1.2960191836 & 1.2960191835 & $1.0000000827 \mathrm{E}-10$ & 1.2940262028 & $1.9929808000 \mathrm{E}-03$ \\
\hline $\mathbf{1 . 2}$ & 1.3266517673 & 1.3266517673 & $0.0000000000 \mathrm{E}+00$ & 1.3244127386 & $2.2390287000 \mathrm{E}-03$ \\
\hline $\mathbf{1 . 3}$ & 1.3579558136 & 1.3579558136 & $0.0000000000 \mathrm{E}+00$ & 1.3554627016 & $2.4931120000 \mathrm{E}-03$ \\
\hline $\mathbf{1 . 4}$ & 1.3899435370 & 1.3899435371 & $1.0000000827 \mathrm{E}-10$ & 1.3871881920 & $2.7553450000 \mathrm{E}-03$ \\
\hline $\mathbf{1 . 5}$ & 1.4226272553 & 1.4226272552 & $9.9999786229 \mathrm{E}-11$ & 1.4196014162 & $3.0258391000 \mathrm{E}-03$ \\
\hline $\mathbf{1 . 6}$ & 1.4560193830 & 1.4560193830 & $0.0000000000 \mathrm{E}+00$ & 1.4527146827 & $3.3047003000 \mathrm{E}-03$ \\
\hline $\mathbf{1 . 7}$ & 1.4901324269 & 1.4901324268 & $1.0000000827 \mathrm{E}-10$ & 1.4865403962 & $3.5920307000 \mathrm{E}-03$ \\
\hline $\mathbf{1 . 8}$ & 1.5249789787 & 1.5249789787 & $0.0000000000 \mathrm{E}+00$ & 1.5210910520 & $3.8879267000 \mathrm{E}-03$ \\
\hline $\mathbf{1 . 9}$ & 1.5605717097 & 1.5605717097 & $0.0000000000 \mathrm{E}+00$ & 1.5563792300 & $4.1924797000 \mathrm{E}-03$ \\
\hline $\mathbf{2 . 0}$ & 1.5969233630 & 1.5969233630 & $0.0000000000 \mathrm{E}+00$ & 1.5924175880 & $4.5057750000 \mathrm{E}-03$ \\
\hline
\end{tabular}

Table 4. Comparison of results (ARK5, Problem 3)

\begin{tabular}{|l|c|l|l|l|l|}
\hline $\boldsymbol{x}$ & $y_{\text {exact }}$ & $y_{\text {approx [ARK4] }}$ & \multicolumn{1}{|c|}{ Error $_{\text {ARK4 }}$} & $y_{\text {approx [5] }}$ & Error $_{[5]}$ \\
\hline $\mathbf{0 . 0}$ & 1.0000000000 & 1.0000000000 & $0.0000000000 \mathrm{E}+00$ & 1.0000000000 & $0.0000000000 \mathrm{E}+00$ \\
\hline $\mathbf{0 . 1}$ & 1.1099504938 & 1.1099505002 & $6.4000000854 \mathrm{E}-09$ & 1.1099505093 & $1.5499999950 \mathrm{E}-08$ \\
\hline $\mathbf{0 . 2}$ & 1.2392304845 & 1.2392304978 & $1.3299999990 \mathrm{E}-08$ & 1.2392426822 & $1.2197700000 \mathrm{E}-05$ \\
\hline $\mathbf{0 . 3}$ & 1.3862780491 & 1.3862780713 & $2.2200000060 \mathrm{E}-08$ & 1.3863124522 & $3.4403100000 \mathrm{E}-05$ \\
\hline $\mathbf{0 . 4}$ & 1.5489125293 & 1.5489125612 & $3.1900000197 \mathrm{E}-08$ & 1.5489776613 & $6.5132000000 \mathrm{E}-05$ \\
\hline $\mathbf{0 . 5}$ & 1.7247448714 & 1.7247449111 & $3.9699999954 \mathrm{E}-08$ & 1.7248460043 & $1.0113290000 \mathrm{E}-04$ \\
\hline $\mathbf{0 . 6}$ & 1.9114877049 & 1.9114877494 & $4.4499999907 \mathrm{E}-08$ & 1.9116266171 & $1.3891220000 \mathrm{E}-04$ \\
\hline $\mathbf{0 . 7}$ & 2.1071247279 & 2.1071247745 & $4.6599999859 \mathrm{E}-08$ & 2.1073004252 & $1.7569730000 \mathrm{E}-04$ \\
\hline $\mathbf{0 . 8}$ & 2.3099668871 & 2.3099669335 & $4.6400000286 \mathrm{E}-08$ & 2.3101765998 & $2.0971270000 \mathrm{E}-04$ \\
\hline $\mathbf{0 . 9}$ & 2.5186414056 & 2.5186414508 & $4.5200000187 \mathrm{E}-08$ & 2.5188814589 & $2.4005330000 \mathrm{E}-04$ \\
\hline $\mathbf{1 . 0}$ & 2.7320508076 & 2.7320508509 & $4.3300000030 \mathrm{E}-08$ & 2.7323172355 & $2.6642790000 \mathrm{E}-04$ \\
\hline $\mathbf{1 . 1}$ & 2.9493242009 & 2.9493242421 & $4.1199999856 \mathrm{E}-08$ & 2.9496131242 & $2.8892330000 \mathrm{E}-04$ \\
\hline $\mathbf{1 . 2}$ & 3.1697715604 & 3.1697715994 & $3.9000000118 \mathrm{E}-08$ & 3.1700793883 & $3.0782790000 \mathrm{E}-04$ \\
\hline $\mathbf{1 . 3}$ & 3.3928449536 & 3.3928449906 & $3.6999999953 \mathrm{E}-08$ & 3.3931684726 & $3.2351900000 \mathrm{E}-04$ \\
\hline $\mathbf{1 . 4}$ & 3.6181073013 & 3.6181073363 & $3.5000000231 \mathrm{E}-08$ & 3.6184436957 & $3.3639440000 \mathrm{E}-04$ \\
\hline $\mathbf{1 . 5}$ & 3.8452078799 & 3.8452079131 & $3.3200000082 \mathrm{E}-08$ & 3.8455547171 & $3.4683720000 \mathrm{E}-04$ \\
\hline $\mathbf{1 . 6}$ & 4.0738633754 & 4.0738634069 & $3.1499999942 \mathrm{E}-08$ & 4.0742185720 & $3.5519660000 \mathrm{E}-04$ \\
\hline $\mathbf{1 . 7}$ & 4.3038433133 & 4.3038433432 & $2.9899999809 \mathrm{E}-08$ & 4.3042050957 & $3.6178240000 \mathrm{E}-04$ \\
\hline $\mathbf{1 . 8}$ & 4.5349588662 & 4.5349588948 & $2.8599999702 \mathrm{E}-08$ & 4.5353257301 & $3.6686390000 \mathrm{E}-04$ \\
\hline $\mathbf{1 . 9}$ & 4.7670542374 & 4.7670542646 & $2.7199999586 \mathrm{E}-08$ & 4.7674249097 & $3.7067230000 \mathrm{E}-04$ \\
\hline $\mathbf{2 . 0}$ & 5.0000000000 & 5.0000000260 & $2.6000000375 \mathrm{E}-08$ & 5.0003734053 & $3.7340530000 \mathrm{E}-04$ \\
\hline
\end{tabular}

In Tables 1 and 2, the proposed ARK4 method displayed lesser errors than the methods of [3] and [5]. In Tables 3 and 5, the proposed ARK5 method displayed lesser errors than the methods of [7] and [5]. The results of Tables $1-4$ revealed that the proposed Almost Runge-Kutta methods of orders 4 and 5 exhibit negligible errors in relation to the exact solutions. They also performed better than the corresponding existing methods they are compared to by exhibiting lesser errors.

\section{Conclusion}

By the foregoing, it is instructive that the proposed Almost Runge-Kutta methods of orders 4 and 5 do not only exhibit efficiency and reliability, as evident by their respective inconsequential errors in relation to the exact solutions, but also perform better than the existing methods. 


\section{REFERENCES}

[1] Butcher J. C., General linear methods, Computer Mathematics Application. 31, 105-112 (1996).

[2] Butcher J. C., An introduction to Almost Runge-Kutta methods, Applied Numerical Mathematics. 24, 331-342 (1997).

[3] Rattenbury N., Almost Runge-kutta methods for stiff and non-stiff problems, Ph.D. Thesis University of Auckland, New Zealand. Pp. 35, 57 (2005).

[4] Ndanusa A., An optimal 6 step implicit linear multistep method for initial value problems, Master's Thesis, Federal University of Technology Minna, Nigeria. Pp. 64 (2004).

[5] Abraham O., Development of some new classes of explicit Almost Runge-Kutta methods for non-stiff differential equations, Ph.D. Thesis, Federal University of Technology Minna, Nigeria. Pp. 52 (2010).

[6] J. C. Butcher, Numerical Methods for Ordinary Differential Equations, 2nd ed. Chichester, UK. John Wiley \& Sons, Ltd., 2008, ch. 2, pp. 116.

[7] Alimi O. K., On the performance of Richardson extrapolation technique in estimating local truncation errors for explicit Almost Runge-Kutta methods, Master's Thesis, Federal University of Technology, Minna, Nigeria. Pp. 41 (2014).

\section{AUTHORS' BIOGRAPHY}

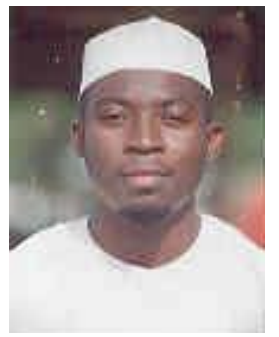

Dr Abdulrahman Ndanusa, obtained a $\mathrm{PhD}$ in Applied Mathematics from Federal University of Technology, Minna, Nigeria in 2012. He is a Senior Lecturer in the Department of Mathematics at Federal University of Technology, Minna, Nigeria where he teaches various courses to undergraduate and postgraduate students. He is an active researcher in Numerical Analysis and Biomathematics where he has several publications in international peer- reviewed journals.

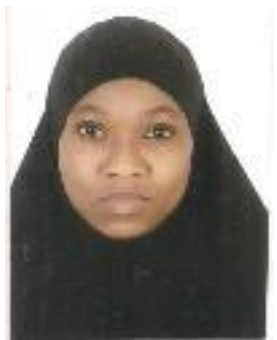

Khadeejah James Audu, holds a Master's degree in Mathematics from Federal University of Technology, Minna, Nigeria (2014). Her research interest is basically in Numerical Analysis. She is currently preparing to enroll for a $\mathrm{PhD}$ programme in Applied Mathematics. 